\title{
O CONCEITO DE INDÚSTRIA 4.0 E OS PRINCIPAIS DESAFIOS DE SUA IMPLANTAÇÃO NO BRASIL
}

\author{
THE INDUSTRY 4.0 CONCEPT AND THE MAIN CHALLENGES OF ITS \\ IMPLEMENTATION IN BRAZIL
}

Ana Cristina Pinheiro Vello - anacrisvello@ gmail.com

Carlos Rodrigo Volante - carlos.volante@ fatectq.edu.br Faculdade de Tecnologia de Taquaritinga (FATEC) -SP -Brasil

DOI: 10.31510/infa.v16i2.686

\begin{abstract}
RESUMO
A indústria mundial vem sofrendo grandes mudanças desde o desenvolvimento dos parques fabris e a tecnologia está cada vez mais presente nela. Neste contexto surgiu a indústria 4.0 ou quarta revolução industrial que tem como alguns de seus pilares os sistemas ciber-físicos $(C P S)$ e a Internet das Coisas (IoT). O objetivo deste artigo é apresentar os conceitos da "Indústria 4.0" e verificar quais são os desafios principais para implantá-la, em especial no Brasil. A metodologia adotada neste trabalho foi a pesquisa bibliográfica que possibilitou abordar a definição de vários autores acerca do tema além da elaboração de um quadro que reuniu trabalhos de autores brasileiros onde foram evidenciados alguns dos desafios das empresas brasileiras ao implantar ou tentar implantar algumas tecnologias trazidas pelo novo modelo de indústria. Foi possível considerar que, no Brasil, a carência de mão de obra especializada, o apoio do governo com políticas estratégicas e a falta infraestrutura tecnológica são os mais apontados.
\end{abstract}

Palavras-chave: Indústria 4.0. Desafios. Brasil. Mão de Obra especializada.

\begin{abstract}
World industry has been undergoing major changes since the development of factory parks and technology is increasingly present in it. In this context came the industry 4.0 or fourth industrial revolution that has as its pillars the cyber-physical systems (CPS) and the Internet of Things (IoT). The purpose of this paper is to present the concepts of "Industry 4.0" and to verify what are the main challenges to implement it, especially in Brazil. The methodology adopted in this work was the bibliographic research that made it possible to approach the definition of several authors on the subject besides the elaboration of a framework that brought together works by Brazilian authors where some of the challenges of Brazilian companies were evidenced when implanting or trying to deploy some technologies brought by the author. New industry model. It was possible to consider that, in Brazil, the lack of specialized labor, government support with strategic policies and the lack of technological infrastructure are the most pointed..
\end{abstract}

Keywords: Industry 4.0. Challenges. Brazil. Skilled labor. 


\section{INTRODUÇÃO}

O presente trabalho tem a finalidade de compartilhar informações sobre a indústria brasileira, que, devido à última crise econômica, tem apresentado quedas em seus níveis de produtividade, conforme Brasil ([2017]). Frente ao cenário industrial brasileiro e do contexto mundial justifica-se à relevância do entendimento de uma nova era da indústria, a chamada indústria 4.0. Sua dinâmica apresenta-se contrária às outras revoluções observado que as empresas e os países devem adotar estratégias e políticas a fim de estarem prontos para as mudanças que ela anuncia, onde inicialmente são vistas como ameaças mas que podem ser fontes de oportunidades de transformação estrutural e desenvolvimento (JOÃO FURTADO, 2017).

Diante desse contexto no qual as organizações se prontificam a buscar sua inserção no ambiente da Indústria 4.0, surge o questionamento que é base para o problema de pesquisa deste estudo: "Quais os desafios que o Brasil encontra para implantar a indústria 4.0?"

A partir dessa questão, o objetivo deste artigo é apresentar os conceitos da indústria 4.0 e verificar quais são os principais desafios de sua implantação no Brasil. De acordo com a revisão bibliográfica fora possível identificar algumas barreiras à implantação desta indústria. Para Confederação Nacional da Indústria - CNI (2016b), o baixo conhecimento por parte das empresas, alto custo de implantação, qualificação do trabalhador e apoio do governo são algumas delas.

Ainda neste trabalho será demonstrada a ligação entre instituições de ensino, governo e empresas para promover a aplicação da indústria 4.0 para perto e, segundo A Voz da Indústria ([2017]), parcerias a favor da Manufatura Avançada - termo também utilizado para referir-se à indústria 4.0 -, podem acontecer entre vários setores e tornar a implantação das tecnologias mais eficiente, reduzir o prazo para os resultados além de proporcionar custos consideravelmente menores. O método de pesquisa adotado no presente artigo foi a revisão bibliográfica com finalidade de analisar dados publicados em materiais e documentos sobre o tema, pertinentes às questões abordadas neste trabalho onde foram levantados resultados pertinentes ao problema de pesquisa. 


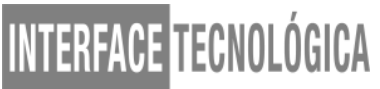

\section{FUNDAMENTAÇÃO TEÓRICA}

A produção industrial mundial passou por revoluções e nos tempos atuais o mundo encontra-se na quarta revolução industrial, a indústria 4.0. No trabalho de Hermann, Otto e Pentek (2015) “como o próprio termo também não está claro, as empresas estão lutando quando se trata de identificação e implementação de cenários Indústria 4.0”.

\subsection{Revoluções Industriais}

A Primeira Revolução Industrial, conforme os autores Brito (2017) e A Voz da Indústria (2018b), aconteceu inicialmente na Inglaterra, compreendida entre os anos de 1760 e 1840 com o desenvolvimento da máquina a vapor utilizada na indústria têxtil. Neste contexto ocorreu o aumento da produção através do início dos parques fabris, substituindo assim os trabalhos artesanais. O uso da tecnologia para navios e trens, a descoberta do telégrafo e do carvão, como fonte de energia, também foram marcos das mudanças sociais e econômicas à época.

Com início nos Estados Unidos da América, a Segunda Revolução Industrial ocorreu entre os anos de 1850 e 1950 e logo chegou a outros países como: França, Alemanha, Itália, Bélgica e Holanda. Seus marcos foram: descoberta do petróleo como fonte de energia, a eletricidade, as usinas hidrelétricas, novos produtos químicos, substituição do ferro pelo aço, produção industrial em série e a divisão do trabalho. A Terceira Revolução Industrial também iniciou-se nos Estados Unidos por volta do ano de 1969. Teve como marco inovações na informática e seu uso na área de produção industrial e no setor de consumo, além da biotecnologia, a exploração espacial, a robótica (com máquina que trabalhavam de maneira automática), a genética e utilização de sistemas de produção flexíveis.

No ano de 2011 a Alemanha apresenta pela primeira vez o conceito Indústria 4.0 “Industrie 4.0", iniciando neste momento a quarta revolução industrial, também chamada de Indústria 4.0 ou Manufatura Avançada. Ela está voltada para os sistemas de Produção CiberFísicos, que é a interconexão das etapas de produção, ou seja, uma indústria mais eficiente e que utiliza fontes de energia renováveis que não foram abordadas nas outras revoluções. 


\subsection{Conceituando a Indústria 4.0}

No ano de 2011 surge, na feira de Hannover (Alemanha) o termo Indústria 4.0 a fim de nomear o projeto alemão que promoveria grande salto de competitividade com uso de novas tecnologias no setor da manufatura cujo objetivo foi revigorar o tecido industrial germânico e fortalecer exportações de equipamentos e soluções "inteligentes" (JOÃO FURTADO, 2017).

Para os autores Kagermann, Wahlster e Helbig (2013) os microcomputadores estão mais poderosos, autônomos e agrupados entre si e com a internet, num ambiente sem fio. Esse cenário resulta na aproximação dos mundos físico e virtual, ou seja, o ciberespaço conhecido como sistema ciber-físico (em inglês Cyber-Physical Systems - CPS). Além disso, em 2012, surge um novo protocolo de internet o IPv62 que trouxe recursos suficientes para ativar a rede direta universal de objetos inteligentes através da Internet tornando possíveis os recursos de rede, informações, objetos e pessoas a fim de dar início à Internet das Coisas e Serviços (em inglês, Internet of Things - IoT e Internet of Services - IoS).

$\mathrm{Na}$ industrialização, os conceitos de Internet of Things - IoT e Internet of Services - IoS estão chegando ao ambiente de fabricação. Em essência, na indústria 4.0 a integração técnica do Cyber-Physical Systems - CPS acontecerá nos setores de fabricação e logística e o uso de $I o T$ e $I o S$ em processos industriais. Isso acarreta implicações na cadeia de valor, nos modelos de negócios, nos serviços a jusante e na organização do trabalho (KAGERMANN, WAHLSTER E HELBIG, 2013)

A incorporação da digitalização à atividade industrial resultou no conceito de Indústria 4.0, caracterizada pela integração e controle da produção a partir de sensores e equipamentos conectados nos sistemas ciber-físicos tornando viável o emprego da inteligência artificial. (CONFEDERAÇÃO NACIONAL DA INDÚSTRIA CNI, 2016a). O autor torna explicita a flexibilidade, autonomia e integração da Indústria 4.0 ao dizer que:

Dispositivos localizados em diferentes unidades da empresa, ou mesmo de empresas diferentes, também trocam informações de forma instantânea sobre compras e estoques, permitindo uma otimização logística até então impensável, estabelecendo maior integração também entre os elos de uma cadeia produtiva. O conceito de Indústria 4.0, contudo, vai além da integração dos processos associados à produção e distribuição, envolvendo, também, todas as diversas etapas da cadeia de valor: do desenvolvimento de novos produtos, como projeto, desenvolvimento, testes e até mesmo a simulação das condições de produção, até o pós-venda. (CONFEDERAÇÃO NACIONAL DA INDÚSTRIA - CNI, 2016a) 
$\mathrm{Na}$ Indústria 4.0 os requisitos do cliente são atendidos e mesmo itens exclusivos podem ser apresentar lucros; na produção, podem acontecer alterações de última hora e respostas flexíveis quando ocorrerem interrupções e falhas além de a tomada de decisões ideal tornar-se mais fácil considerando a transparência no processo de fabricação. Este recente conceito de indústria possibilitará novas maneiras de criar valor e novos modelos de negócios e também proporcionará às empresas em fase de arranque e às pequenas empresas a oportunidade de desenvolver e fornecer serviços de maneira competitiva. (KAGERMANN, WAHLSTER E HELBIG, 2013)

De acordo com Fia, (2018) essa revolução 4.0 tem como base alguns princípios e alguns pilares que são premissas para seu desenvolvimento e implantação, são eles:

- Coleta e análise de dados instantaneamente, possibilitando tomada de decisão precisa e rápida;

- Informações Virtuais, através das tecnologias que possibilitam rastrear e monitorar todos os processos remotamente;

- Descentralização do trabalho quando a própria máquina toma decisão de se auto ajustar e fornecendo informações de seus ciclos de trabalho e avaliando necessidades da produção, em tempo real;

- Orientação a serviços, ou seja, softwares orientados a disponibilizarem soluções como serviços, conectados com toda a indústria, conceito de Internet of Services$I o S$;

- Modularidade é o que possibilita a flexibilidade da planta industrial, ou seja, módulos podem ser acoplados e desacoplados de acordo com a demanda da fábrica;

- Interoperabilidade, cuja função é estabelecer comunicação entre máquinas e operações;

- Big data capacidade de armazenar o grande número de dados coletados que servirão de base para tomada de decisão da máquina, entre outros;

- Inteligência artificial, no cenário industrial, é a tomada de decisão da máquina, sem interferência humana, baseada em um banco de dados;

- Computação em nuvem tratam-se de servidores compartilhados ligados através da internet possibilitando a conectividade fora dos limites físicos da empresa. 


\subsection{A Indústria no Brasil}

De acordo com Brasil ([2017]), Indústria representa menos de 10\% do PIB - Produto Interno Bruto e o Brasil ocupa a $69^{\mathrm{a}}$ colocação no Índice Global de Inovação também observouse que entre 2006 os anos de 2016 a produtividade da indústria no Brasil caiu mais de 7\%, assim como no Índice Global de Competitividade da Manufatura, o Brasil caiu da 5a posição em 2010 para a $29^{a}$ posição em 2016.

Ainda segundo o autor, no Relatório "Readiness for the Future of Production Report 2018" (WEF) o Brasil está na 41ª posição referente estrutura de produção e na 47 posição nos vetores de produção da indústria. Porém o Brasil possui potencial para melhorar sua posição nesta nova economia. E em 2018, os indicadores econômicos demonstram recuperação na economia com crescimento do PIB de 2,8\% em 2018 e 3,0\% em 2019 o que irá interferir na indústria brasileira.

O Ministério da Industria, Comércio e Serviços - MDIC compôs, em junho de 2017, o Grupo de Trabalho para a Indústria 4.0 (GTI 4.0), cujo objetivo se deu em elaborar a agenda nacional para o tema. Ele reune 50 instituições entre governo, empresas, sociedade civil organizada, onde ocorreram diversos debates sobre perspectivas e ações para a Indústria 4.0 brasileira. Alguns temas são aumento de competitividade, mudanças nas cadeias produtivas, novo mercado de trabalho, uso de tecnologias digitais, startups.

\subsection{Desafios para implantação da Indústria 4.0 no mundo}

Para Schuh; Gausemeier; Wahlster, (2017) muitas empresas apenas implementam projetos-piloto e estes possuem características parecidas com um estudo de viabilidade tecnológica ao invés de medidas da indústria 4.0. Esses projetos restringem a indústria 4.0 pois eles não conseguem demonstrar todo o potencial dela, ou seja, não observam aspectos essenciais para sua fase de implantação que são a cultura e a estrutura da organização.

Outro aspecto apresentado pelo autor foi que existem falhas na transmissão real dos processos organizacionais ou em considerar reais necessidades de empresas de manufatura. Ainda ressalta que são poucos os exemplos de transformação em empresas individuais e na economia como um todo. 
Segundo Kagermann, Wahlster e Helbig (2013), “atingir a mudança de paradigma necessária para entregar a Indústria 4.0 é um projeto de longo prazo e envolverá um processo gradual.” Os autores ainda ressaltam em sua obra que a implantação da indústria 4.0 trará um desafio tecnológico e também nas implicações da organização, a qual possibilita o desenvolvimento de modelos de negócios e corporativos além de proporcionar maior ligação entre funcionários.

A Alemanha, país que trouxe o termo Industria 4.0 pela primeira vez possui, de acordo com Kagermann, Wahlster e Helbig (2013), boa posição na implantação destes novos conceitos uma vez que o país implantou com sucesso a terceira revolução industrial, gerenciou os impactos na força de trabalho, conta com forte base na indústria de manufatura de softwares, e conhecimento das novas tecnologias. $\mathrm{O}$ apoio do governo também teve parte neste sucesso uma vez que desde 2006 traz a Internet da Coisas - IoT em sua estratégia de alta tecnologia. Ainda assim, os desafios são a aceitação de questões tecnológicas e o número restrito de profissionais qualificados.

\section{PROCEDIMENTOS METODOLÓGICOS}

O procedimento metodológico utilizado no presente artigo foi a pesquisa bibliográfica. Para Gonçalves (2005) a pesquisa bibliográfica "trata-se do primeiro passo em qualquer tipo de pesquisa; sua finalidade é conhecer as diferentes contribuições científicas sobre o assunto que se pretende estudar”. Ainda conforme Gonçalves (2005) a revisão bibliográfica ou revisão de literatura é necessária a fim de promover um levantamento dos trabalhos que existem sobre o assunto assim como conhecer seus autores.

De acordo com Santos (2004, p. 20 apud GONÇALVES, 2005, p. 58), a pesquisa bibliográfica "é aquela que é desenvolvida a partir de material já elaborado, constituído principalmente de livros e artigos científicos [...]". A partir destes conceitos foram analisados diversos artigos, documentos, e-books, e conceitos publicados em revistas, congressos, sites de instituições de ensino, governamentais e de empresas ligadas à indústria e à tecnologia.

A busca em artigos científicos teve como finalidade verificar o conhecimento deste conceito de indústria no Brasil e analisar quais são as dificuldades, já consideradas em estudos sobre o que o país encontra ou encontrou ao implantar alguns conceitos da indústria 4.0.0 Para tanto, foram pesquisados os termos "desafios de implantação da indústria 4.0 no Brasil", 
“indústria 4.0 no Brasil”, “industrialização no Brasil” e afim de conceituar a indústria alguns dos termos de busca foram "conceito de indústria 4.0", "Indústria 4.0”, "Industrie 4.0”, "o que é indústria 4.0 e quais seus impactos”.

\section{RESULTADOS E DISCUSSÃO}

A seguir será apresentado um quadro, elaborado pela autora deste artigo, que tem como objetivo reunir e sintetizar os desafios apontados por diversos autores, referente a implantação de conceitos da indústria 4.0, no Brasil, também chamada de quarta revolução industrial. $\mathrm{O}$ quadro torna possível identificar quais os desafios que foram levantados com maior frequência e compará-los com a teoria trazida na seção de fundamentação teórica, ou seja, elencar quais dificuldades que o país encontra e compará-las com o cenário mundial.

Quadro 1 - Desafios para implantação da indústria 4.0 no Brasil

\begin{tabular}{|l|l|}
\hline Autor & Desafios apontados \\
\hline A Voz Da Indústria ([2017]) & $\begin{array}{l}\text { Desafios práticos nas áreas de convergência } \\
\text { tecnológica; ausência de regulação; dificuldade } \\
\text { com recursos humanos, com cadeias de valor e } \\
\text { infraestrutura tecnológica. }\end{array}$ \\
\hline A Voz Da Indústria (2018a) & $\begin{array}{l}\text { Falta de conhecimento sobre conceitos 4.0; } \\
\text { implantação com altos custos; qualificação dos } \\
\text { trabalhadores; ausência de infraestrutura } \\
\text { tecnológica; falta de incentivos do governo; } \\
\text { inexperiência no processo de transição. }\end{array}$ \\
\hline Brito (2017) & $\begin{array}{l}\text { Poucos setores competitivos; poucos produtos } \\
\text { inovadores. }\end{array}$ \\
\hline Confederação Nacional Da Indústria - CNI & $\begin{array}{l}\text { Escassez de conhecimento por parte das } \\
\text { empresas, alto custo de implantação, carência de } \\
\text { qualificação do trabalhador e apoio do governo. }\end{array}$ \\
\hline $\begin{array}{l}\text { Confederação Nacional Da Indústria - CNI } \\
\text { (2016b) }\end{array}$ & $\begin{array}{l}\text { Integração digital nas cadeias produtivas; } \\
\text { falta de apoio governamental à adoção de novas } \\
\text { tecnologias; escassez de novo modelo de } \\
\text { educação profissional, ausência de regulação; } \\
\text { ausência de políticas industriais; raros } \\
\text { investimentos em equipamentos; falta de } \\
\text { infraestrutura tecnológica. }\end{array}$ \\
\hline Costa et al. (2018) & $\begin{array}{l}\text { Falta de estratégia industrial, tecnologia e mão de } \\
\text { obra especializada. }\end{array}$ \\
\hline Daudt; Willcox (2016) & $\begin{array}{l}\text { Falta de políticas sobre demanda efetiva assim } \\
\text { como de apoios científicos e tecnológicos. }\end{array}$ \\
\hline Fia (2018) & $\begin{array}{l}\text { Serviços e produtos com pouco valor agregado e } \\
\text { com margens de lucro pequenas; qualificar } \\
\text { profissionais; melhorar infraestruturas logísticas } \\
\text { de telecomunicações; falta de conhecimento, por }\end{array}$ \\
\hline
\end{tabular}




\begin{tabular}{|l|l|}
\hline Autor & Desafios apontados \\
\hline Firjan (2016) & $\begin{array}{l}\text { parte da empresa, de conceitos e como mensurar } \\
\text { impactos e benefícios. }\end{array}$ \\
\hline Katayama; Coelho; Seguchi (2018) & $\begin{array}{l}\text { Escassez de políticas estratégicas, incentivos e } \\
\text { fomentos do governo; unir gestores; baixo } \\
\text { desenvolvimento tecnológico; formação de } \\
\text { profissionais qualificados, distância entre } \\
\text { indústria e instituiçôes, acadêmicas e de pesquisa. }\end{array}$ \\
\hline Kupfer (2016) & $\begin{array}{l}\text { Organização não possui autoconhecimento. } \\
\text { Algumas indústrias nacionais ainda estão na } \\
\text { revolução 2.0; defasagens em tecnologias de } \\
\text { informação e comunicação; gargalos regulatórios } \\
\text { e de infraestrutura tecnológica. }\end{array}$ \\
\hline Lima et al. (2018) & $\begin{array}{l}\text { Capacitar bem trabalhador a fim de modernizar a } \\
\text { empresa. }\end{array}$ \\
\hline Roberto Vermulm (2018) & $\begin{array}{l}\text { Tecnologias ainda são pouco conhecidas, } \\
\text { institucionalidade frágil, políticas sem definição } \\
\text { de prioridades; proposição de políticas sem } \\
\text { estabelecimento de instrumentos de ação; falta de } \\
\text { articulação entre instituições do setor público; } \\
\text { falta de cooperação efetiva entre governo e } \\
\text { empresariado industrial. }\end{array}$ \\
\hline Santos; Manhães; Lima (2018) & $\begin{array}{l}\text { Falta de domínio sobre os componentes e } \\
\text { tecnologias; a carência de mão de obra } \\
\text { qualificada; o impacto cultural nas organizações; } \\
\text { análise dos ambientes da empresa. }\end{array}$ \\
\hline
\end{tabular}

Fonte: Elaborado pela autora (2019)

Após analisar o quadro foi possível observar que os desafios frequentemente levantados são a carência de mão de obra qualificada para trabalhar com as novas tecnologias trazidas pelo conceito de indústria 4.0, também observou-se que o apoio do governo é essencial e que no Brasil este ainda é algo que deverá ser aprimorado com políticas estratégicas envolvendo estes novos conceitos. A infraestrutura tecnológica foi outro desafio com grande ocorrência no quadro demonstrando que o país ainda necessita de avanços e desenvolvimento de tecnologia a favor da indústria brasileira. Por fim, o quadro confirma a teoria apresentada na seção de fundamentação teórica.

\section{CONSIDERAÇÕES FINAIS}

O artigo teve como objetivo apresentar os conceitos da indústria 4.0 e identificar quais são os desafios de sua implantação no Brasil. Como método de atingir tal objetivo foi adotada a revisão bibliográfica que possibilitou a elaboração de um quadro com os desafios apontados 
por diversos autores brasileiros e compará-los com os desafios trazidos na fundamentação teórica.

Este trabalho possibilitou considerar que os desafios frequentemente trazidos pelos autores são a qualificação dos trabalhadores, o apoio do governo com políticas estratégicas que envolvam estes novos conceitos e a carência da infraestrutura tecnológica, outro desafio apresentado comprovando que o país deverá investir na superação destes desafios em favor da indústria brasileira.

Como um dos desafios principais trazidos neste trabalho foi a carência de mão de obra especializada, ou seja, a necessidade de os funcionários aprimorarem seus conhecimentos sobre as recentes tecnologias é sugerido que sejam desenvolvidas novas pesquisas a respeito deste tema a fim de favorecer as empresas e ao setor industrial brasileiro como um todo, ou seja, unindo os principais interessados nesta revolução que são as indústrias, o governo e as instituições de ensino, para aumentar a produtividade e competitividade do Brasil frente aos outros países.

\section{REFERÊNCIAS}

A VOZ DA INDÚSTRIA (Brasil). Parceria entre empresas, universidades e startups rumo à Manufatura Avançada: Entenda como a união desses três pilares pode facilitar a modernização da indústria. [2017]. Disponível em: <https://www.feimec.com.br/a-voz-daindustria-materiais/parcerias-manufatura-avancada.html>. Acesso em: 13 jun. 2019.

A VOZ DA INDÚSTRIA (Brasil) $a .5$ desafios da implantação da Industria 4.0: como superá-los? 2018. Indústria 4.0 by TOTVS. Disponível em:

$<$ https://avozdaindustria.com.br/desafios-implantacao-industria-4-0-como-superar/>. Acesso em: 15 jun. 2019.

A VOZ DA INDÚSTRIA (Brasil) $b$. Caminho até a indústria 4.0: os destaques das revoluções industriais. 2018. Indústria 4.0 by TOTVS. Disponível em: <https://avozdaindustria.com.br/industria-4-0-os-destaques-das-revolucoes-industriais/>. Acesso em: 05 jun. 2019.

BRASIL. Agência Brasileira de Desenvolvimento Industrial - ABDI. Ministério da Indústria Comércio e Serviços - Governo Federal. Agenda brasileira para a Indústria 4.0: O Brasil preparado para os desafios do futuro. [2017]. Apoio: FGV - Projetos. Disponível em: <http://www.industria40.gov.br/>. Acesso em: 06 jun. 2019.

BRITO, Alexandra Antonia Freitas de. A Quarta Revolução Industrial e as Perspectivas para o Brasil. Revista Científica Multidisciplinar Núcleo do Conhecimento, [s.1.], v. 2, n. 7 , p.91-96, out. 2017. Disponível em: 
<https://www.nucleodoconhecimento.com.br/administracao/quarta-revolucaoindustrial\#_ftn1>.Acesso em: 12 ago. 2019.

CONFEDERAÇÃO NACIONAL DA Indústria - CNI (Brasília) b. SONDAGEM ESPECIAL 66: Indústria 4.0. 2016. Disponível em:

<http://www.portaldaindustria.com.br/estatisticas/sondesp-66-industria-4-0/>. Acesso em: 16 ago. 2019.

CONFEDERAÇÃO NACIONAL DA INDÚSTRIA CNI (Brasília) a. Desafios para a indústria 4.0 no Brasil. 2016. Disponível em:

$<$ https://www.portaldaindustria.com.br/publicacoes/2016/8/desafios-para-industria-40-nobrasil/>. Acesso em: 06 jun. 2019.

COSTA, Indianara Mikaele da et al. AUTOMÓVEIS HÍBRIDOS NA INDÚSTRIA 4.0 E NO BRASIL. 2018. Disponível em:

<http://sienpro.catalao.ufg.br/up/1012/o/99._AUTOM\%C3\%93VEIS_H\%C3\%8DBRIDOS_ NA_IND\%C3\%9ASTRIA_4.0_E_NO_BRASIL.pdf>. Acesso em: 05 jun. 2019.

DAUDT, Gabriel Marino; WILLCOX, Luiz Daniel. Reflexões críticas a partir das experiências dos Estados Unidos e da Alemanha em manufatura avançada. 2016. Disponível em: <https://web.bndes.gov.br/bib/jspui/handle/1408/9936>. Acesso em: 14 jun. 2019.

FIA, Fundação Instituto de Administração. Indústria 4.0: o que é, consequências, impactos positivos e negativos [Guia Completo]. 2018. Disponível em:

<https://fia.com.br/blog/industria-4-0/>. Acesso em: 06 jun. 2019.

FIRJAN (Brasil). Indústria 4.0. 2016. Elaboração do Documento: DIN - Diretoria de Inovação GIE - Gerência de Inovação Estratégica. Disponível em:

<https://www.firjan.com.br/publicacoes/publicacoes-de-inovacao/industria-4-0-1.htm>. Acesso em: 10 ago. 2019.

GONÇALVES, Hortência de Abreu. Manual de Metodologia da Pesquisa Científica. São Paulo: Avercamp, 2005. 142 p.

HERMANN, Mario; OTTO, Boris; PENTEK, Tobias. Design Principles for Industrie 4.0 Scenarios: A Literature Review. 2015. Disponível em:

$<$ https://www.researchgate.net/publication/307864150_Design_Principles_for_Industrie_40_ Scenarios_A_Literature_Review>. Acesso em: 06 ago. 2019.

JOÃO FURTADO (Brasil). Instituto de Estudos Para O Desenvolvimento Industrial Iedi. Indústria 4.0: A Quarta Revolução Industrial e os desafios para a Indústria e para o desenvolvimento brasileiro. 2017. Disponível em:

<https://iedi.org.br/artigos/top/estudos_comercio/20170721_iedi_industria_4_0.html>. Acesso em: 06 ago. 2019.

KAGERMANN, Henning; WAHLSTER, Wolfgang; HELBIG, Johannes. Recommendations for implementing the strategic initiative INDUSTRIE 4.0: Final report of the Industrie 4.0 
Working Group. 2013. Acatech - National Academy of Science and Engineering. Disponível em: <https://en.acatech.de/publication/recommendations-for-implementing-the-strategicinitiative-industrie-4-0-final-report-of-the-industrie-4-0-working-group/>. Acesso em: 04 jun. 2019.

KATAYAMA, Mari Tomita; COELHO, João Carlos Martins; SEGUCHI, Henrique Jun Muramatsu. Gestão avançada como fundamento para a implantação de conceitos da indústria 4.0. 2018. Disponível em:

<http://sienpro.catalao.ufg.br/up/1012/o/GEST\%C3\%83O_AVAN\%C3\%87ADA_COMO_F UNDAMENTO_PARA_A_IMPLANTA\%C3\%87\%C3\%830_DE_CONCEITOS_DA_IND \%C3\%9ASTRIA_4.0.pdf>. Acesso em: 02 jun. 2019.

KUPFER, David. Indústria 4.0 Brasil. 2016. Disponível em:

<https://www.valor.com.br/opiniao/4661797/industria-40-brasil>. Acesso em: 11 jun. 2019.

LIMA, Fernanda Pereira et al. A gestão do conhecimento no contexto da indústria 4.0. 2018. Disponível em:

<http://sienpro.catalao.ufg.br/up/1012/o/143._A_GEST\%C3\%83O_DO_CONHECIMENTO_ NO_CONTEXTO_DA_IND\%C3\%9ASTRIA_4.0.pdf>. Acesso em: 05 jun. 2019.

ROBERTO VERMULM (Brasil). Instituto de Estudos Para O Desenvolvimento Industrial Iedi. Políticas Para O Desenvolvimento Da Indústria 4.0 No Brasil. 2018. Disponível em: <https://web.bndes.gov.br/bib/jspui/handle/1408/15486>. Acesso em: 10 ago. 2019.

SANTOS, Marcos; MANHÃES, Aline Martins; LIMA, Angélica Rodrigues. Indústria 4.0: Desafios e oportunidades para o Brasil. 2018. Disponível em:

<https://ri.ufs.br/bitstream/riufs/10423/2/Industria_4_0.pdf>. Acesso em: 12 ago. 2019.

SCHUH, Günther; GAUSEMEIER, Jürgen; WAHLSTER, Wolfgang. Industrie 4.0 Maturity Index: Managing the Digital Transformation of Companies. 2017. Disponível em:

$<$ https://www.acatech.de/wp-

content/uploads/2018/03/acatech_STUDIE_Maturity_Index_eng_WEB.pdf>. Acesso em: 15 maio 2019. 